\title{
Two-dimensional modeling the static parameters for a submicron field-effect transistor
}

\author{
M. Zaabat, M. Draid \\ Institut de Physique Universite de OUM EL BOUAGHI, Algeria
}

\begin{abstract}
A comparison of two different models for simulation of submicron GaAs MESFETs static characteristics has been made. A new two-dimensional numerical model is presented to investigate the submicron field-effect transistor characteristics, the influence of the geometry of the component, like the inter-electrode distance, on the capacities. All simulation revealed the existence of a high contact electric field near the gate, which creates a depopulated zone around the gate, but the preceding studies have neglected the edge effects, which are very significant for the submicron MESFETs.
\end{abstract}

Keywords: MESFET, submicron device simulation, modeling.

Manuscript received 27.02.09; revised manuscript received 16.06.09; accepted for publication 10.09.09; published online 30.11.09.

\section{Introduction}

GaAs metal-semiconductor field-effect transistors (MESFETs) are widely used for microwave and digital applications because of their superior high frequency characteristics [1-4]. Simulation of GaAs-based nonlinear circuits necessitates the use of an accurate MESFET model, if the predicted and achieved circuit performances are closely correlated. In device fabrication, one usually starts with a computer simulation of the device or the circuit to be made.

The development or improvement of new dies of components requires means for the modeling, realization and characterization. It is thus very significant to predetermine the characteristic of the component, physical modeling finds here one of its principal applications. Taking into account the complexity of operation of a field-effect transistor to the submicron gate, according to the application chosen, the optimization of the component according to the geometrical and physical parameters is not possible by only an experimental approach $[2,8]$. It is imperative to understand the phenomena, which exist, the variation of only one technological parameter being able to have various consequences on the characteristics and possibilities of the field-effect transistor. Those are not easily analyzable without a precise determination of the internal parameters that govern them $[3,4]$.

The designer of the components must know the influence of the technological parameters, so that he can envisage their influence on the behavior of the device, in particular when it is a question of monolithic integrated microwave circuit [5-8].
The output characteristics of a GaAs MESFET can be simulated by using two modeling techniques: 1) numerical models and 2) physical models. Considered in this paper is a two-dimensional model based on an analytical solution of the Poisson equation.

This paper investigates the gate-bias dependence of a field-effect transistor to the gate submicron depth of the depopulated zone. The influence of the edge effects on the profile of the depopulated zone as well as capacities and charge distribution is investigated.

We have made two-dimensional simulation of the component with field effects considering the effect of the edge and the real position (source, gate, and drain) of our structure (Fig. 1). Since then there have been a number of theoretical papers, but a lot of them uses the one-dimensional modeling [3, 9].

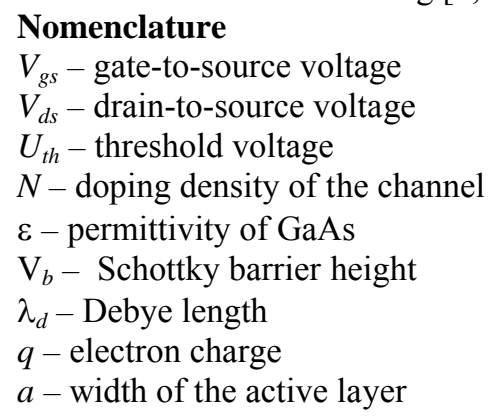

\section{Analytical model}

Fig. 1 shows a normal planar field-effect transistor simulated in this study. We use the analytical model determined by [2], this model is based on the Green function: 


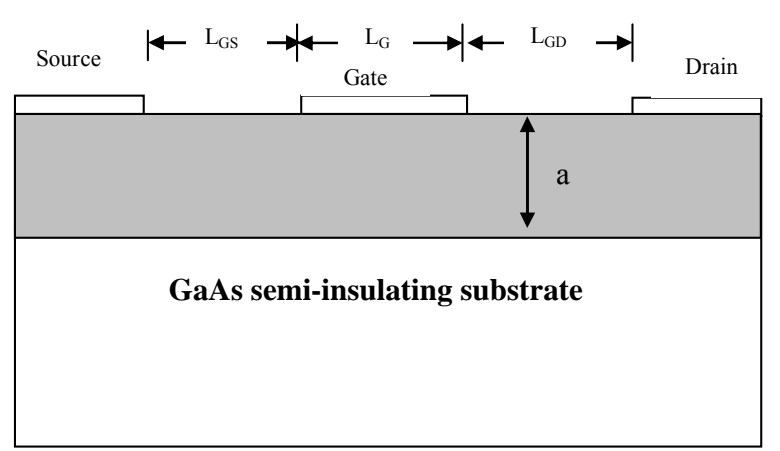

Fig. 1. The structure of the flied-effect transistor.

$G\left(x / x_{0}, y / y_{0}\right)$

where $(x, y)$ - point of observation

$\left(x_{0}, y_{0}\right)$ - observed point.

\subsection{Shockley approximation}

The Shockley approximation allows to express the width of the depletion layer through $V_{g s}$ and $V_{d s}$

$h_{s}=a \sqrt{\frac{V_{b i}-V_{g s}}{V_{p}}}$,

where $h_{s}$ is the depth of the depletion layer on the side of source. $h_{d}=a \sqrt{\frac{V_{b i}-V_{g s}+V_{d s}}{V_{p}}}$,

where $h_{d}$ is the depth of the depletion layer on the side of drain.

And

$V_{p}=U_{t}$

\subsection{The potential}

Poisson's equation in all space if considering the quasi-static mode is given by:

$\operatorname{div}[\varepsilon(x, y) \cdot \operatorname{grad}(V(x, y))]=-\rho(x, y)$

with $\rho(x, y)=\left\{\begin{array}{cc}\rho_{s} & \text { on metal } \\ \rho_{0} & \text { in ZD } \\ 0 & \text { otherwise }\end{array}\right.$

and $\varepsilon(x, y)=\left\{\begin{array}{cc}\varepsilon_{0} & \text { in vacuum } \\ \varepsilon_{s} & \text { in SC }\end{array}\right.$

\subsection{Simulation}

Fig. 3 shows the simulation program.

- The potential $V$ represents the applied potential $V_{G}$ plus the potential of barrier $V_{b}$.

- Calculation of the limit of the depopulated zone is made by combining the equation (3) with the following hypotheses:

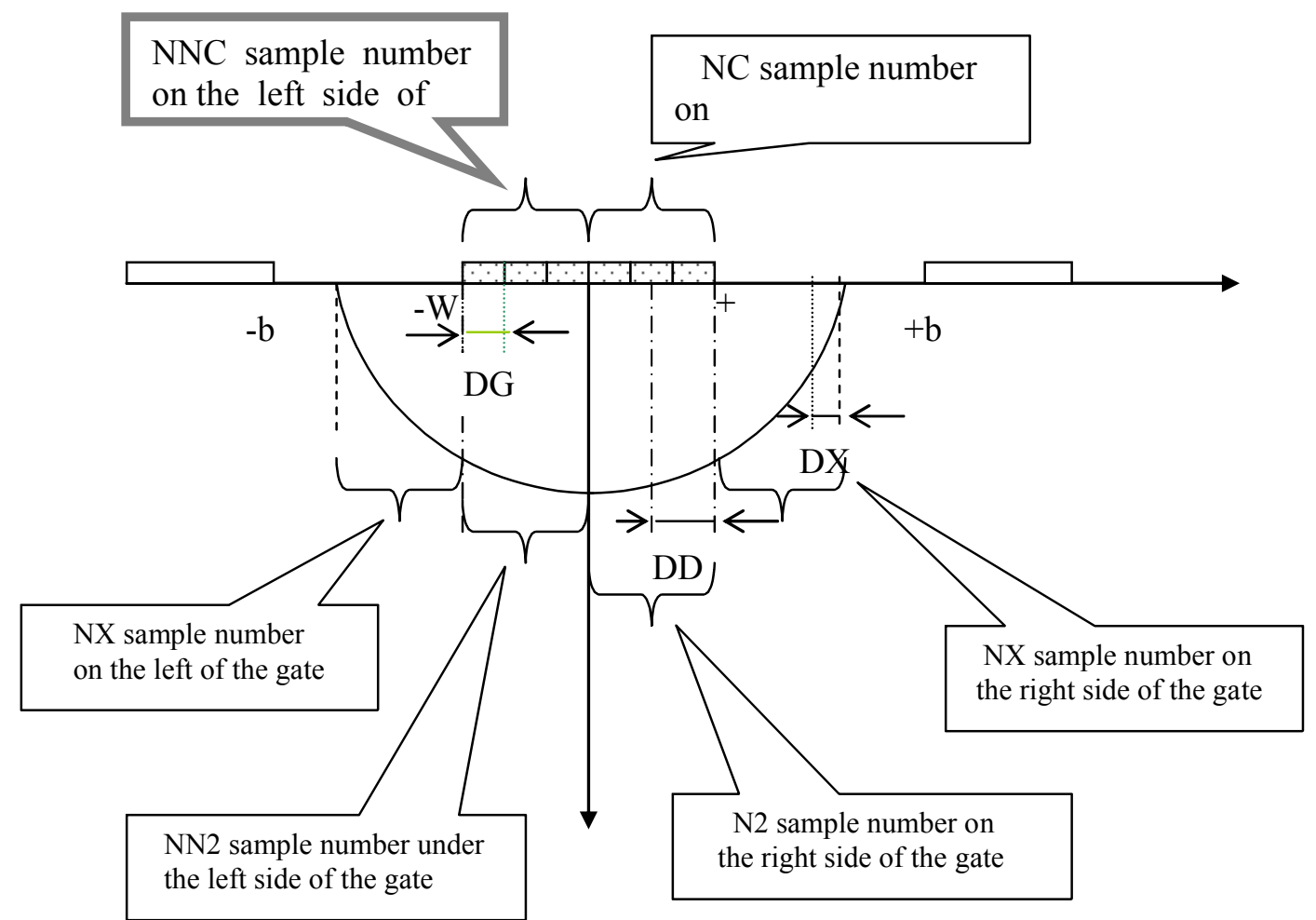

Fig. 2. Scheme of discretization of the structure. 


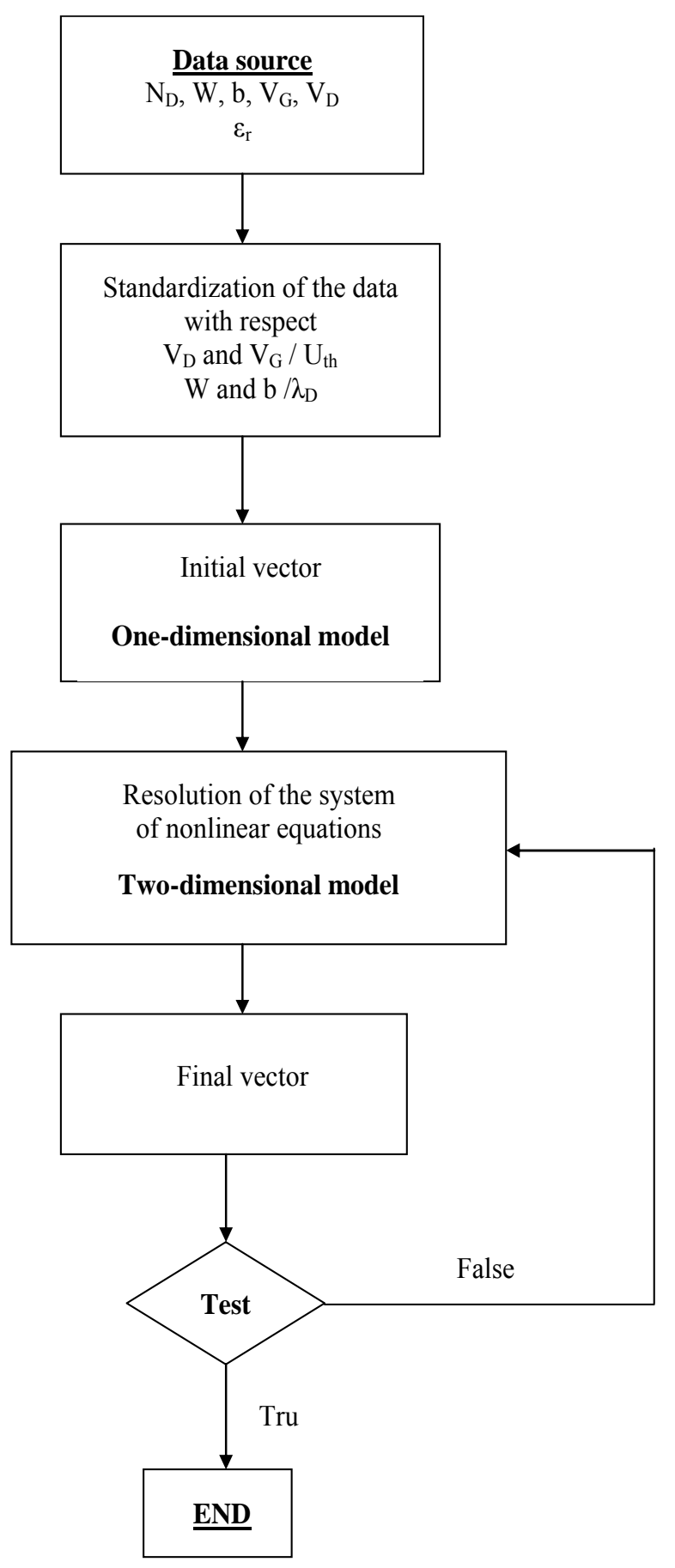

Fig. 3. The simulation program.

$-V(x, y)=V$ on the metal conductor,

$-V(x, y)=0$ on the profile of the depopulated zone.

It results in a system of non-linear equations. Resolving them by the method of moments (Fig. 3) allows to know the depopulated zone profile in the discredited form as well as the electric charge distribution.

We use an initial vector determined by the onedimensional approximation by Shockley (2), (3).

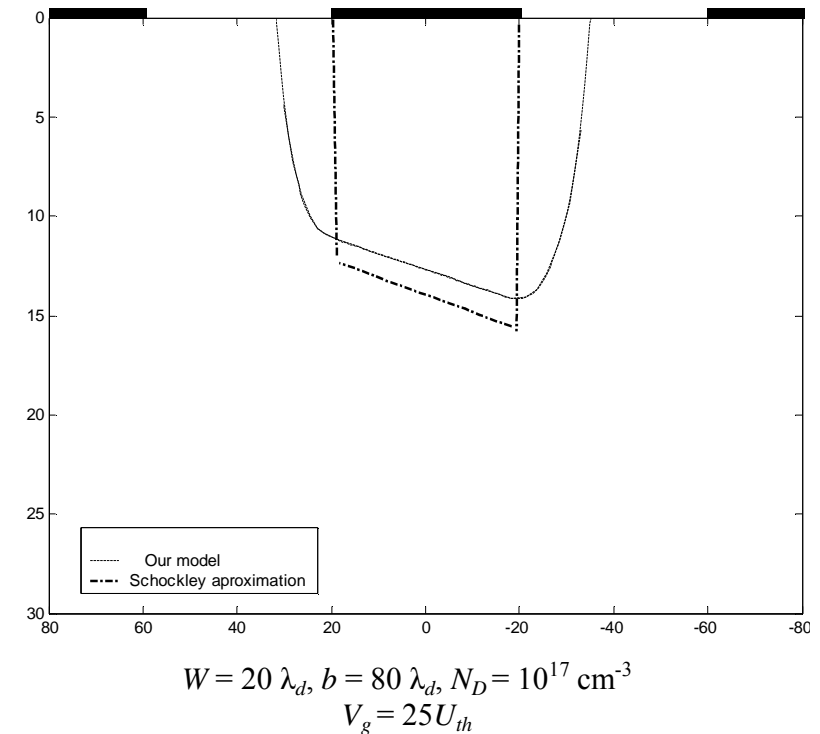

Fig. 4. Depth Y of the depletion layer.

\section{Results}

The observed characteristics along with the results simulated using our model and the Schokley model are shown in Figs 4, 5, 6 and 7. Variation of the depopulated zone with gate bias determined by our model has been compared with those given by the one dimensional model. Fig. 7 shows variation of the gate bias with the charge density. So, the Shockley approximation neglecting the edge effect that is taken in our model we show in Figs 4, 5 and 6.

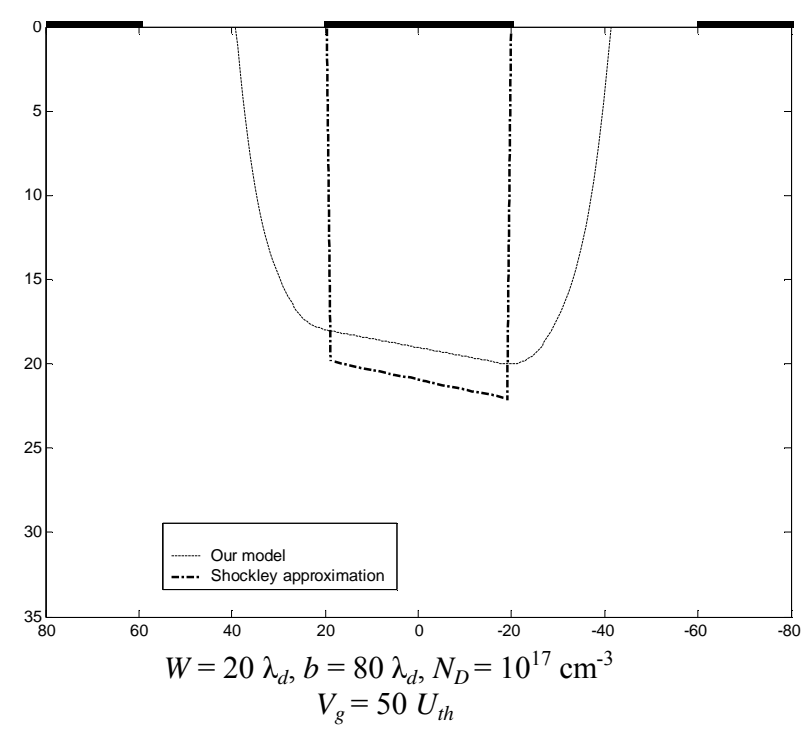

Fig. 5. Depth Y of the depletion layer. 


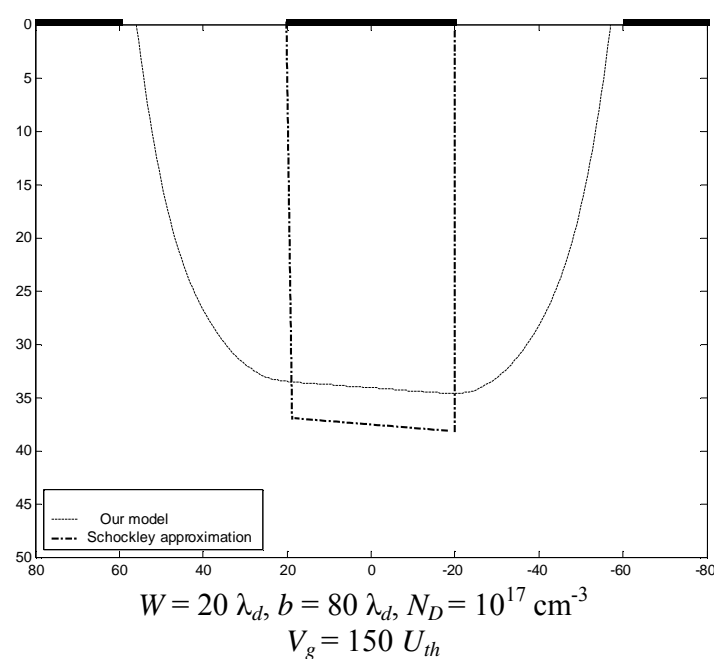

Fig. 6. Depth Y of the depletion layer.

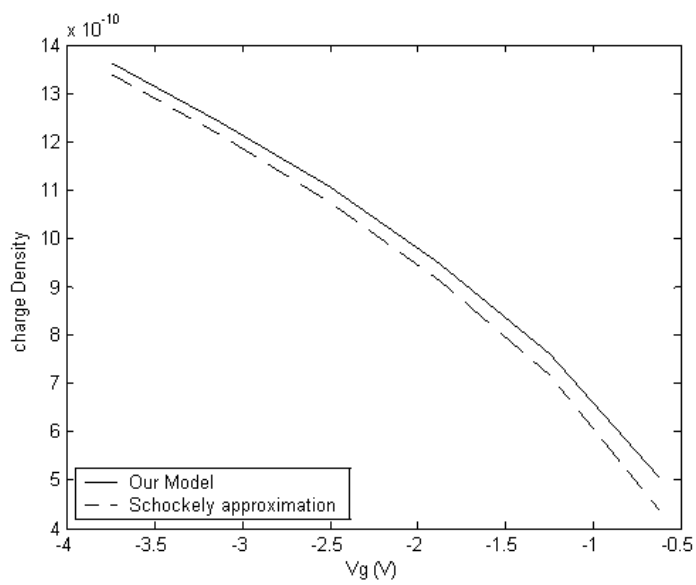

Fig. 7. Variation of the gate bias with the charge density.

\section{Conclusion}

This algorithm has been developed for simulation of static characteristics in submicron MESFETs. Simulation which we have realized gives variation in the height of the channel or the thickness of the depopulated zone with variation of the voltage applied to the gate, and thus allows to control the current across the component when taking into account of exact geometry of the component. Then, we proved that the onedimensional $[3,9,10]$ model gives the results with a very low precision, especially for the high gate bias. We can use our results for determining the static characteristics for the field effect transistor (like capacities, resistance, conductance of channel, ...). We think that our results can contribute to designing the field-effect transistors in planar technology, and to consequent reducing the manufacturing cost of the component.

\section{References}

1. P. Kennis and L. Faucon, Rigorous analysis of planar MIS transmission lines layered lossy media // Electron. Lett. 18(14), July 1982.

2. A. Benghalia, M. Ahmadpanah and H. Baudrand, Accurate two-dimensional approach for capacitance calculation in microcoplanar MES transmission lines // Electron. Lett. 24(16), p. 996998 (August 1988).

3. M.J. Golio, J.R.C. Golio // IEEE Trans. Microwave Theory Tech. 39, 142-146 (1991).

4. T. Enoki, S. Sugitani, Y. Yamane // IEEE Trans. Electron. Devices 37, p. 935-941 (1990).

5. S. Lepaul, Contribution a la modelisation numerique des composant electroniques aux dimension nanometrique // These de doctorat, Unversite de Paris VI, 1996.

6. A. Cappy and W. Heinrich, High frequency FET noise performance: a new approach // IEEE Trans. Electron. Devices, ED-36, no. 2 (Feb. 1989).

7. H. Fujimoto \& Al, New high power planar gate GaAs MESFET with improved gate-drain breakdown voltage // Electron. Lett. 61(2), p. 137 (Jan. 1995).

8. Kazushige Horio and Tomiko Yamada, Towdimensional analysis of surface state effects on turn-on characteristics in GaAs MESFET's // IEEE Trans. Electron. Devices, ED-46, no. 4 (April 1999).

9. W. Schokley and W.T. Read, Statistics of the recombination of holes and electrons // Phys. Rev. 87, p. 835-842 (1952).

10. M.M. Ahmed // IEEE Trans. Electron. Devices 47, p. 299-303 (2000). 